\title{
Simulation of Vehicle Maintenance on the Basis of Functional Resonance Analysis Method using Function-Oriented Technology
}

\author{
Olha Sakno ${ }^{1, *}$, Tetiana Kolesnikova ${ }^{1}$, Vasyl Ollo ${ }^{2}$ \\ 1 Prydniprovs'ka State Academy of Civil Engineering and Architecture, Faculty of Mechanics, 24A Chernyshevsky Street, Dnipro, 49600, Ukraine; \\ E-Mails: sakno-olga@ukr.net \\ 2 Military Academy (Odesa), Department of Food and Supplies, 10 Fontanska doroga, Odesa, 65009, Ukraine; E-Mails: ollovp@gmail.com
}

\begin{abstract}
In the article, using the functional resonance analysis method, the process of traffic safety control of vehicles that carry out cargo delivery and transportation of passengers, when their technical condition changes during operation, is simulated. On the basis of statistical data, the reasons of the change of the technical condition of the chassis of the vehicles and their influence on its operational properties were analysed. Resilience Decision Support (Resilience DS) software is used to solve this problem. Such a tool made it possible to simulate the sociotechnical system "Person-Car-Organization of maintenance and repair system" and to form the formal models for constant evaluation of the effective functioning of the system of maintenance of vehicles and conditions of increasing their operation. To develop a maintenance model using a functional resonance analysis method, data was collected at a proprietary vehicle maintenance station. The model consists of a set of functions that determine the actions executed during maintenance of vehicles. The use of function-oriented technologies in a vehicle maintenance system allows simulation and maximizing overall operational parameters of vehicle due to local increase in technical capabilities and properties of individual elements, surfaces and/or zones of a part, depending on the functional features of operation.
\end{abstract}

Keywords: vehicle maintenance, technical condition, functional resonance analysis method, function-oriented technology

\section{Introduction}

Successful management of the transport process can be implemented by identifying the influence of factors on the effectiveness of its implementation and determining the patterns of occurrence of causes that can negatively affect it. The issues of monitoring the safety of the movement of vehicles during the implementation of the processes of cargo delivery and passenger transportation are relevant and require their solution using modern scientific methods. The functional resonance analysis method (FRAM) is used to study complex sociotechnical systems. It was created and described by Eric Hollnagel, who noted that complex technical systems contain a large number of subsystems and components, the variability of which is usually absorbed by the system with minimal impact on the overall system. The main sources of this variability are people, technology and organization, which ensures the serviceable state of the technique.

Professor Eric Hollnagel stated that, since these elements are not linearly connected to each other, they can lead to the accidents. When the variations of the components become too large for the system to absorb, then the result becomes unfavourable 
or random. This concerns the functional resonance effect arising from a situation where the system is not able to function in normal operation due to changes in its daily productivity.

A model of the FRAM describes the ability of the functions of the system components of a vehicle to resonate and create hazards during its operation that can get out of control and cause accidents or accidents due to the low level of functioning of the vehicle maintenance and repair system.

The FRAM [1] supports a system analysis process aimed at identifying interdependencies and system behaviours that are potentially important for a tool which focuses on the interdependence of the process and its dynamics. This is a method of modeling complex organizational and technical systems obtained by the theory of sustainable health [2], which relates to how to achieve success of the technical system by adapting it to difficult conditions [3]. Recent documents have shown the use of FRAM to understand the legal implementation of guidelines of management the efforts to achieving security [4]. The method of functional resonance analysis involves the identification of functions (technological, human or organizational activities) in the in the day-to-day operation of a technical system [5-7].

The purpose of the paper is to simulate, on the basis of the FRAM, the process of control over the safety of the vehicle movement in a result of changes of its technical condition, which affects the deterioration of its steerability, stability and smoother running characteristics during operation.

Cross-functional process can be considered in the implementation with involving of multiple systems at the same time at the upper level. In terms of system integrators methodology [8-9], it is these processes finally realize the Product Lifecycle Management (PLM) systems. It is a good idea to use such a process hidden greatest potential to improve the quality of engineering products. Any complex hierarchical organization, reaching a certain size, has a problem that the local criteria of individual subsystems begin to dominate to ensure extreme values of the global objective function [10].

\section{General vehicular principles of the functional resonance analysis method}

The activity of the FRAM is to record the influence of the technical condition of the chassis of the vehicle on the change in its operational properties and traffic safety from a system point of view.

Using the FRAM takes a systematic perspective, which means that the analysis cannot be limited to a certain part of the socio-technical system, but it should consider a broader picture when the organization of the control process is considered as a whole, and not as maintenance and assembly of components of the vehicle's chassis [11-13]. The method analyses the organization of the traffic safety control process as a socio-technical system, where the technology is embedded in the social context and develops, tests, launches and maintains a serviceable technical condition of the vehicle.

\section{The method is based on four basic principles:}

1. Equivalence principle:operability and inoperability are equivalent to the fact that they both stem from shift work and the functioning of the system as a whole. There are no special reasons that only work for failures.

2. Approximate adjustment principle: variability, as a way to adapt a person to the management of the maintenance and repair system and to the operating conditions of the vehicle. People (specialists) always know how to adjust what they do to fit the situation (traffic safety). Such variability of efficiency is inevitable, ubiquitous, and necessary.

3. Principle of surprise: the appearance of operability and disability is not a direct result of variability within a particular task or function, but rather is a combination of the variability of many functions. The variability of the functioning of the system may combine unexpectedly, which leads to disproportionate large results (non-linear effects). The result arises if it cannot be attributed to the functions of the system or explained by them.

4. Functional resonance principle: unexpected "enhanced" effects of interactions between different sources of variability underlie the phenomenon, which is described by functional resonance. Functional resonance is a detection signal (it can be detected) that comes out from an unintended combination of the variability of many signals. Functional resonance is an alternative to linear causality.

Models of FRAM can be used to study potential sources of variability by simulating and identifying context-sensitive aspects of the functioning of the system as a whole, such as human, technological, and organizational. This approach supports the assessment of the potential of the system for solving variability, taking into account the expected and unexpected variability, and follows from the work of the technical system (vehicle as a whole).

The basic unit of analysis is the functional hexagon (Figure 1). Using six aspects of each function (time, control, output, resources, preconditions and 


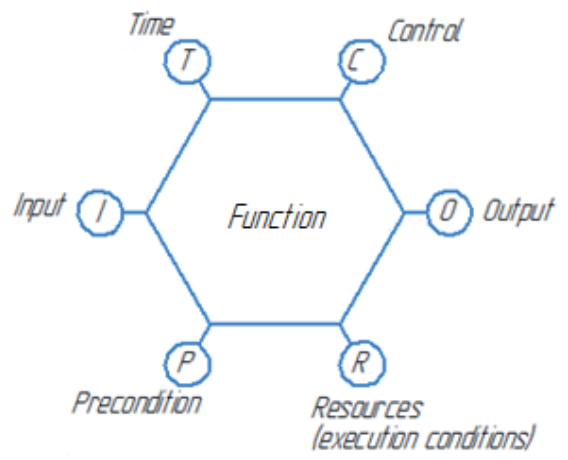

Figure 1: FRAM Function.

input), system interactions aimed at identifying potential sources of resonance are studied.

Thus, the output of the function can be an input, a prerequisite, or even a forced control aspect of one or another function of the system (Table 1). This process can lead to the identification of possible sources of attenuation for undesirable variability. For example, if the resources for a certain function are evaluated as "more than necessary", this may indicate the existence of a "spare system", which can act as a damper barrier to ensure the safety of the vehicle. Research process of possible connections between functions in order to identify both potential undesirable sources of variability and barriers is called an instance of FRAM (i.e., direct proximity).

\section{Simulation of a vehicle maintenance system}

To achieve this goal, the technical support of Resilience DS software is used [14]. Such a tool allows you to simulate the socio-technical system "Person-Car-Organization of maintenance and

Table 1: Characteristics of FRAM functional units- repair system" and form formal models to constantly evaluate the effectiveness of the functioning of the system of technical maintenance and repair of vehicles and improve their operating conditions.

Data collection for simulate by FRAM was carried out in three different stages. At the first stage, in the spring of 2019, a two-day visit to the technical service station in the city Dnipro was organized, where technical maintenance of branded vehicles, in particular passenger cars, is carried out. During the visit, there was made the familiarization of the process of organization the maintenance of vehicles in specific conditions. The second stage of data collection was to conduct a seminar, which was attended by researchers, a contact person and employees involved in the process of preparation and execution vehicles maintenance. The purpose of the seminar was to discuss, revise and update the previous FRAM model, developed on the basis of information collected during a visit to the branded technical service station and official documentation. The seminar helped researchers identify some of the functions of the system and determine their aspects (input, output, control, preconditions, time and resources to ensure the serviceable technical condition of vehicles).

The collected data was used to develop by FRAM of a vehicles maintenance model, consisting of a set of functions that describe the actions taken to their service and to ensure their serviceable technical condition.

In our study, to ensure the safe movement of a vehicle during its operation, it is necessary, without fail, to conduct periodic technical impact on its chassis, which consists in: diagnostics; checking the condition of shock absorbers, springs, support cups, brake blocks, discs, hoses, backlash in ball bearings

\begin{tabular}{|l|l|l|}
\hline Parameter & Symbol & Description \\
\hline Input & $I$ & $\begin{array}{l}\text { Input information (or input data) that characterizes a function in the system's functioning. } \\
\text { These are links to previous functions. Input data runs the execution or action of a function. }\end{array}$ \\
\hline Output & $T$ & $\begin{array}{l}\text { The result of the function (output). Provides links to further functions. Represents the result } \\
\text { of changing the technical condition of the system elements (vehicle). }\end{array}$ \\
\hline Control & $C$ & $\begin{array}{l}\text { The time, which is required for processing (preparation, diagnosis, maintenance of system } \\
\text { elements) by functional subsection or subsystem. }\end{array}$ \\
\hline Preconditions & $P$ & $\begin{array}{l}\text { Limitations, methods and procedures of control. They determine how the function tran- } \\
\text { smits technical data and/or is monitored. }\end{array}$ \\
\hline Resources & $R$ & $\begin{array}{l}\text { System conditions which determine the conditions of functioning and which must be } \\
\text { provided before function execution. }\end{array}$ \\
\hline
\end{tabular}


Acta Mechanica Slovaca

Journal published by Faculty of Mechanical Engineering - Technical University of Košice

Table 2: Characteristics of FRAM functional units for vehicles maintenance.

\begin{tabular}{|c|c|c|c|c|c|c|c|c|}
\hline Number in fig. 4 & F-Function & Description & I-Input & 0-Output & C-Control & P-Preconditions & R-Resources & T-Time \\
\hline \multirow{3}{*}{1} & \multirow{3}{*}{ Vehicle } & \multirow{3}{*}{$\begin{array}{l}\text { Design features } \\
\text { of vehicle }\end{array}$} & $\begin{array}{l}\text { Reference information } \\
\text { of automobile plant }\end{array}$ & $\begin{array}{l}\text { Technical } \\
\text { condition of } \\
\text { vehicle }\end{array}$ & $\begin{array}{l}\text { Diagnostic } \\
\text { parameters }\end{array}$ & $\begin{array}{l}\text { Running conditions } \\
\text { of vehicle }\end{array}$ & $\begin{array}{l}\text { Vehicle } \\
\text { Maintenance }\end{array}$ & $\begin{array}{l}\text { Run before next } \\
\text { service }\end{array}$ \\
\hline & & & Reliability of vehicle & $\begin{array}{l}\text { Maintenance } \\
\text { schedule }\end{array}$ & - & - & - & - \\
\hline & & & Cost of vehicle & $\begin{array}{l}\text { Diagnostic } \\
\text { parameters }\end{array}$ & - & - & - & - \\
\hline \multirow[t]{2}{*}{2} & \multirow{2}{*}{$\begin{array}{l}\text { Stability of } \\
\text { vehicle }\end{array}$} & \multirow{2}{*}{$\begin{array}{l}\text { Running ability } \\
\text { of vehicle }\end{array}$} & $\begin{array}{l}\text { Technical condition of } \\
\text { vehicle }\end{array}$ & $\begin{array}{l}\text { Reliability of } \\
\text { vehicle }\end{array}$ & $\begin{array}{l}\text { Procedure of } \\
\text { automobile plant }\end{array}$ & Design of vehicle & $\begin{array}{l}\text { Technical } \\
\text { condition of } \\
\text { running gear }\end{array}$ & $\begin{array}{l}\text { Run before next } \\
\text { service }\end{array}$ \\
\hline & & & - & $\begin{array}{l}\text { Maintenance } \\
\text { schedule }\end{array}$ & $\begin{array}{l}\text { Technical } \\
\text { maintenance }\end{array}$ & $\begin{array}{l}\text { Vehicle } \\
\text { Maintenance }\end{array}$ & - & - \\
\hline \multirow{2}{*}{3} & \multirow{2}{*}{$\begin{array}{l}\text { Steering } \\
\text { response }\end{array}$} & \multirow{2}{*}{$\begin{array}{l}\text { Running ability } \\
\text { of vehicle }\end{array}$} & $\begin{array}{l}\text { Technical condition of } \\
\text { vehicle }\end{array}$ & $\begin{array}{l}\text { Reliability of } \\
\text { vehicle }\end{array}$ & $\begin{array}{l}\text { Procedure of } \\
\text { automobile plant }\end{array}$ & Design of vehicle & $\begin{array}{l}\text { Technical } \\
\text { condition of } \\
\text { running gear }\end{array}$ & $\begin{array}{l}\text { Run before next } \\
\text { service }\end{array}$ \\
\hline & & & - & $\begin{array}{l}\text { Maintenance } \\
\text { schedule }\end{array}$ & $\begin{array}{l}\text { Technical } \\
\text { maintenance }\end{array}$ & $\begin{array}{l}\text { Vehicle } \\
\text { Maintenance }\end{array}$ & $\begin{array}{l}\text { Technical } \\
\text { condition of } \\
\text { steering }\end{array}$ & $\begin{array}{l}\text { Running } \\
\text { conditions of } \\
\text { vehicle }\end{array}$ \\
\hline \multirow{2}{*}{4} & \multirow{2}{*}{$\begin{array}{l}\text { Safety of } \\
\text { traffic }\end{array}$} & \multirow{2}{*}{$\begin{array}{l}\text { Running ability } \\
\text { of vehicle }\end{array}$} & $\begin{array}{l}\text { Technical condition of } \\
\text { vehicle }\end{array}$ & $\begin{array}{l}\text { Rule Of The } \\
\text { Road }\end{array}$ & $\begin{array}{l}\text { Procedure of } \\
\text { automobile plant }\end{array}$ & Design of vehicle & $\begin{array}{l}\text { Technical } \\
\text { condition of } \\
\text { vehicle }\end{array}$ & $\begin{array}{l}\text { Run before next } \\
\text { service }\end{array}$ \\
\hline & & & - & $\begin{array}{l}\text { Maintenance } \\
\text { schedule }\end{array}$ & - & $\begin{array}{l}\text { Vehicle } \\
\text { Maintenance }\end{array}$ & $\begin{array}{l}\text { Technical } \\
\text { maintenance }\end{array}$ & $\begin{array}{l}\text { Running } \\
\text { conditions of } \\
\text { vehicle }\end{array}$ \\
\hline \multirow{3}{*}{5} & \multirow{3}{*}{ Fuel efficiency } & \multirow{3}{*}{$\begin{array}{l}\text { Running ability } \\
\text { of vehicle }\end{array}$} & $\begin{array}{l}\text { Technical condition of } \\
\text { vehicle }\end{array}$ & $\begin{array}{l}\text { Fuel con- } \\
\text { sumption }\end{array}$ & $\begin{array}{l}\text { Procedure of } \\
\text { automobile plant }\end{array}$ & Design of vehicle & $\begin{array}{l}\text { Fuel and } \\
\text { velocity engine } \\
\text { performance }\end{array}$ & $\begin{array}{l}\text { Run before next } \\
\text { service }\end{array}$ \\
\hline & & & Maintenanceschedule & - & - & $\begin{array}{l}\text { Vehicle } \\
\text { Maintenance }\end{array}$ & $\begin{array}{l}\text { Technical } \\
\text { condition of } \\
\text { vehicle }\end{array}$ & $\begin{array}{l}\text { Running } \\
\text { conditions of } \\
\text { vehicle }\end{array}$ \\
\hline & & & - & - & - & - & $\begin{array}{l}\text { Technical } \\
\text { maintenance }\end{array}$ & - \\
\hline \multirow{2}{*}{6} & \multirow{2}{*}{$\begin{array}{l}\text { Technical } \\
\text { condition of } \\
\text { running gear }\end{array}$} & \multirow{2}{*}{$\begin{array}{l}\text { Compliance } \\
\text { with the design } \\
\text { and regulatory } \\
\text { documentation } \\
\text { of an automo- } \\
\text { bile plant }\end{array}$} & $\begin{array}{l}\text { Technical condition of } \\
\text { vehicle }\end{array}$ & $\begin{array}{l}\text { Procedure of } \\
\text { automobile } \\
\text { plant }\end{array}$ & $\begin{array}{l}\text { Diagnostic } \\
\text { parameters }\end{array}$ & Design of vehicle & $\begin{array}{l}\text { Vehicle } \\
\text { Maintenance }\end{array}$ & $\begin{array}{l}\text { Run before next } \\
\text { service }\end{array}$ \\
\hline & & & Maintenance schedule & - & - & $\begin{array}{l}\text { Running conditions } \\
\text { of vehicle }\end{array}$ & $\begin{array}{l}\text { Technical } \\
\text { maintenance }\end{array}$ & - \\
\hline \multirow[b]{2}{*}{7} & \multirow{2}{*}{$\begin{array}{l}\text { Technical } \\
\text { condition of } \\
\text { tired wheel }\end{array}$} & \multirow{2}{*}{$\begin{array}{l}\text { Compliance } \\
\text { with the design } \\
\text { and regulatory } \\
\text { documentation } \\
\text { of an automo- } \\
\text { bile plant }\end{array}$} & $\begin{array}{l}\text { Technical condition of } \\
\text { vehicle }\end{array}$ & $\begin{array}{l}\text { Fuel con- } \\
\text { sumption }\end{array}$ & $\begin{array}{l}\text { Diagnostic } \\
\text { parameters }\end{array}$ & Design of vehicle & $\begin{array}{l}\text { Vehicle } \\
\text { Maintenance }\end{array}$ & $\begin{array}{l}\text { Run before next } \\
\text { service }\end{array}$ \\
\hline & & & Maintenance schedule & $\begin{array}{l}\text { Procedure of } \\
\text { automobile } \\
\text { plant }\end{array}$ & - & $\begin{array}{l}\text { Running conditions } \\
\text { of vehicle }\end{array}$ & $\begin{array}{l}\text { Technical } \\
\text { maintenance }\end{array}$ & - \\
\hline \multirow[t]{3}{*}{8} & \multirow{3}{*}{$\begin{array}{l}\text { Technical } \\
\text { condition } \\
\text { of tire }\end{array}$} & \multirow{3}{*}{$\begin{array}{l}\text { Compliance } \\
\text { with the design } \\
\text { and regulatory } \\
\text { documentation } \\
\text { of an automo- } \\
\text { bile plant }\end{array}$} & Maintenance schedule & $\begin{array}{l}\text { Reference } \\
\text { information } \\
\text { of automobile } \\
\text { plant }\end{array}$ & Tread pattern & $\begin{array}{l}\text { Running conditions } \\
\text { of vehicle }\end{array}$ & $\begin{array}{l}\text { Vehicle } \\
\text { Maintenance }\end{array}$ & $\begin{array}{l}\text { Run before next } \\
\text { service }\end{array}$ \\
\hline & & & $\begin{array}{l}\text { Technical condition of } \\
\text { vehicle }\end{array}$ & Tire wear & - & - & $\begin{array}{l}\text { Technical } \\
\text { maintenance }\end{array}$ & - \\
\hline & & & Diagnostic parameters & - & - & - & - & - \\
\hline
\end{tabular}




\begin{tabular}{|c|c|c|c|c|c|c|c|c|}
\hline \multirow{2}{*}{9} & \multirow{2}{*}{$\begin{array}{l}\text { Technical } \\
\text { condition of } \\
\text { engine }\end{array}$} & \multirow{2}{*}{$\begin{array}{l}\text { Compliance } \\
\text { with the design } \\
\text { and regulatory } \\
\text { documentation } \\
\text { of an automo- } \\
\text { bile plant }\end{array}$} & Fuel consumption & $\begin{array}{l}\text { Fuel and } \\
\text { velocity engine } \\
\text { performance }\end{array}$ & $\begin{array}{l}\text { Procedure of } \\
\text { automobile plant }\end{array}$ & $\begin{array}{l}\text { Vehicle Mainte- } \\
\text { nance }\end{array}$ & $\begin{array}{l}\text { Technical } \\
\text { maintenance }\end{array}$ & $\begin{array}{l}\text { Run before next } \\
\text { service }\end{array}$ \\
\hline & & & $\begin{array}{l}\text { Run before next } \\
\text { service }\end{array}$ & - & - & - & - & - \\
\hline \multirow{3}{*}{10} & \multirow{3}{*}{$\begin{array}{l}\text { Running gear } \\
\text { diagnostics }\end{array}$} & \multirow{3}{*}{$\begin{array}{l}\text { Vehicle } \\
\text { Maintenance }\end{array}$} & Diagnostic parameters & $\begin{array}{l}\text { Reference } \\
\text { information } \\
\text { of automobile } \\
\text { plant }\end{array}$ & Rule Of The Road & $\begin{array}{l}\text { Run before next } \\
\text { service }\end{array}$ & 1-2 mechanics & 2-3hours \\
\hline & & & $\begin{array}{l}\text { Technical condition of } \\
\text { running gear }\end{array}$ & $\begin{array}{l}\text { Prevention } \\
\text { planning }\end{array}$ & - & $\begin{array}{l}\text { Vehicle } \\
\text { Maintenance }\end{array}$ & - & - \\
\hline & & & - & - & - & $\begin{array}{l}\text { Technical condition } \\
\text { of vehicle }\end{array}$ & - & - \\
\hline \multirow[t]{2}{*}{11} & \multirow{2}{*}{$\begin{array}{l}\text { Checkup } \\
\text { shocks, } \\
\text { springs } \\
\text { and end } \\
\text { abutment }\end{array}$} & \multirow[t]{2}{*}{$\begin{array}{l}\text { Vehicle } \\
\text { Maintenance }\end{array}$} & \multirow[t]{2}{*}{ Prevention planning } & $\begin{array}{l}\text { Reference } \\
\text { information } \\
\text { of automobile } \\
\text { plant }\end{array}$ & $\begin{array}{l}\text { Procedure of } \\
\text { automobile plant }\end{array}$ & $\begin{array}{l}\text { Technical condition } \\
\text { of running gear }\end{array}$ & 1-2 mechanics & 1-2 hours \\
\hline & & & & $\begin{array}{l}\text { Preventive } \\
\text { repair }\end{array}$ & - & $\begin{array}{l}\text { Vehicle } \\
\text { Maintenance }\end{array}$ & - & - \\
\hline \multirow[t]{2}{*}{12} & \multirow{2}{*}{$\begin{array}{l}\text { Checking } \\
\text { brake block, } \\
\text { brake disk } \\
\text { and brake } \\
\text { hose }\end{array}$} & \multirow[t]{2}{*}{$\begin{array}{l}\text { Vehicle } \\
\text { Maintenance }\end{array}$} & Prevention planning & $\begin{array}{l}\text { Reference } \\
\text { information } \\
\text { of automobile } \\
\text { plant }\end{array}$ & $\begin{array}{l}\text { Procedure of } \\
\text { automobile plant }\end{array}$ & $\begin{array}{l}\text { Technical condition } \\
\text { of running gear }\end{array}$ & 1-2 mechanics & 1-2 hours \\
\hline & & & - & $\begin{array}{l}\text { Preventive } \\
\text { repair }\end{array}$ & - & $\begin{array}{l}\text { Vehicle Mainte- } \\
\text { nance }\end{array}$ & - & - \\
\hline \multirow[t]{2}{*}{13} & \multirow[t]{2}{*}{$\begin{array}{l}\text { Scanning } \\
\text { for steering } \\
\text { wheel play }\end{array}$} & \multirow[t]{2}{*}{$\begin{array}{l}\text { Vehicle } \\
\text { Maintenance }\end{array}$} & Prevention planning & $\begin{array}{l}\text { Reference } \\
\text { information } \\
\text { of automobile } \\
\text { plant }\end{array}$ & $\begin{array}{l}\text { Procedure of } \\
\text { automobile plant }\end{array}$ & $\begin{array}{l}\text { Technical condition } \\
\text { of running gear }\end{array}$ & 1-2 mechanics & 1-2 hours \\
\hline & & & - & $\begin{array}{l}\text { Preventive } \\
\text { repair }\end{array}$ & - & $\begin{array}{l}\text { Vehicle } \\
\text { Maintenance }\end{array}$ & - & - \\
\hline \multirow[t]{2}{*}{14} & \multirow{2}{*}{$\begin{array}{l}\text { Checkup } \\
\text { silentblocks } \\
\text { and other } \\
\text { components } \\
\text { of a running } \\
\text { gear }\end{array}$} & \multirow[t]{2}{*}{$\begin{array}{l}\text { Vehicle } \\
\text { Maintenance }\end{array}$} & Prevention planning & $\begin{array}{l}\text { Reference } \\
\text { information } \\
\text { of automobile } \\
\text { plant }\end{array}$ & $\begin{array}{l}\text { Procedure of } \\
\text { automobile plant }\end{array}$ & $\begin{array}{l}\text { Technical condition } \\
\text { of running gear }\end{array}$ & 1-2 mechanics & 1-2 hours \\
\hline & & & - & $\begin{array}{l}\text { Preventive } \\
\text { repair }\end{array}$ & - & $\begin{array}{l}\text { Vehicle } \\
\text { Maintenance }\end{array}$ & - & - \\
\hline \multirow[t]{2}{*}{15} & \multirow{2}{*}{$\begin{array}{l}\text { Checking, } \\
\text { control and } \\
\text { change } \\
\text { a wheel } \\
\text { bearing }\end{array}$} & \multirow[t]{2}{*}{$\begin{array}{l}\text { Vehicle } \\
\text { Maintenance }\end{array}$} & Prevention planning & $\begin{array}{l}\text { Reference } \\
\text { information } \\
\text { of automobile } \\
\text { plant }\end{array}$ & $\begin{array}{l}\text { Procedure of } \\
\text { automobile plant }\end{array}$ & $\begin{array}{l}\text { Technical condition } \\
\text { of running gear }\end{array}$ & 1-2 mechanics & 1-2 hours \\
\hline & & & - & $\begin{array}{l}\text { Preventive } \\
\text { repair }\end{array}$ & - & $\begin{array}{l}\text { Vehicle } \\
\text { Maintenance }\end{array}$ & - & - \\
\hline \multirow[t]{2}{*}{16} & \multirow{2}{*}{$\begin{array}{l}\text { Diagnosis of } \\
\text { the condition } \\
\text { of alignment } \\
\text { condition }\end{array}$} & \multirow[t]{2}{*}{$\begin{array}{l}\text { Vehicle } \\
\text { Maintenance }\end{array}$} & Prevention planning & $\begin{array}{l}\text { Reference } \\
\text { information } \\
\text { of automobile } \\
\text { plant }\end{array}$ & $\begin{array}{l}\text { Procedure of } \\
\text { automobile plant }\end{array}$ & $\begin{array}{l}\text { Technical condition } \\
\text { of running gear }\end{array}$ & 1-2 mechanics & 1-2 hours \\
\hline & & & - & $\begin{array}{l}\text { Preventive } \\
\text { repair }\end{array}$ & - & $\begin{array}{l}\text { Vehicle } \\
\text { Maintenance }\end{array}$ & - & - \\
\hline \multirow[t]{2}{*}{17} & \multirow[t]{2}{*}{$\begin{array}{l}\text { Checkup an } \\
\text { automobile } \\
\text { tire }\end{array}$} & \multirow[t]{2}{*}{$\begin{array}{l}\text { Vehicle } \\
\text { Maintenance }\end{array}$} & Prevention planning & $\begin{array}{l}\text { Reference } \\
\text { information } \\
\text { of automobile } \\
\text { plant }\end{array}$ & Rule Of The Road & $\begin{array}{l}\text { Technical condition } \\
\text { of running gear }\end{array}$ & 1 mechanic & 1 hour \\
\hline & & & - & $\begin{array}{l}\text { Preventive } \\
\text { repair }\end{array}$ & - & $\begin{array}{l}\text { Vehicle } \\
\text { Maintenance }\end{array}$ & - & - \\
\hline
\end{tabular}




\begin{tabular}{|c|c|c|c|c|c|c|c|c|}
\hline \multirow{3}{*}{18} & \multirow{3}{*}{ Tread life } & \multirow{3}{*}{$\begin{array}{l}\text { Vehicle } \\
\text { Maintenance }\end{array}$} & $\begin{array}{l}\text { Reference information } \\
\text { of automobile plant }\end{array}$ & $\begin{array}{l}\text { Run before next } \\
\text { service }\end{array}$ & - & - & - & - \\
\hline & & & - & $\begin{array}{l}\text { Vehicle } \\
\text { Maintenance }\end{array}$ & - & - & - & - \\
\hline & & & - & Cost of vehicle & - & - & - & - \\
\hline
\end{tabular}

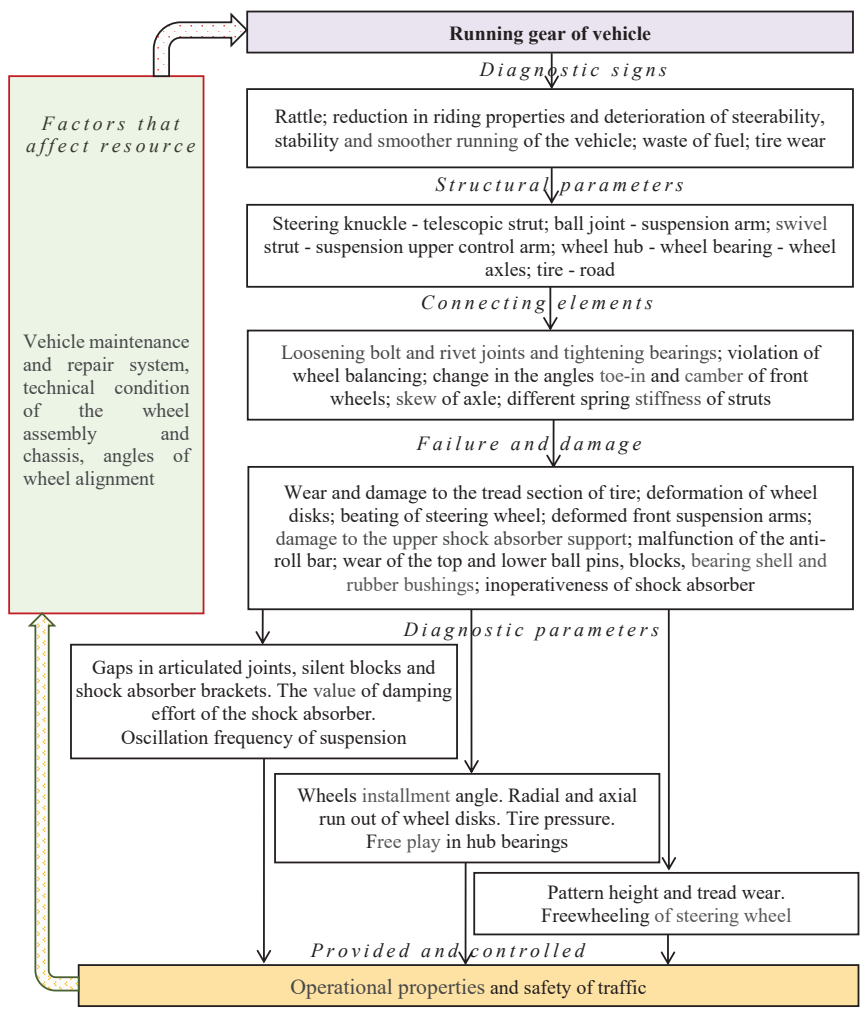

Figure 2: Structural and investigative model of the running gear of a car.

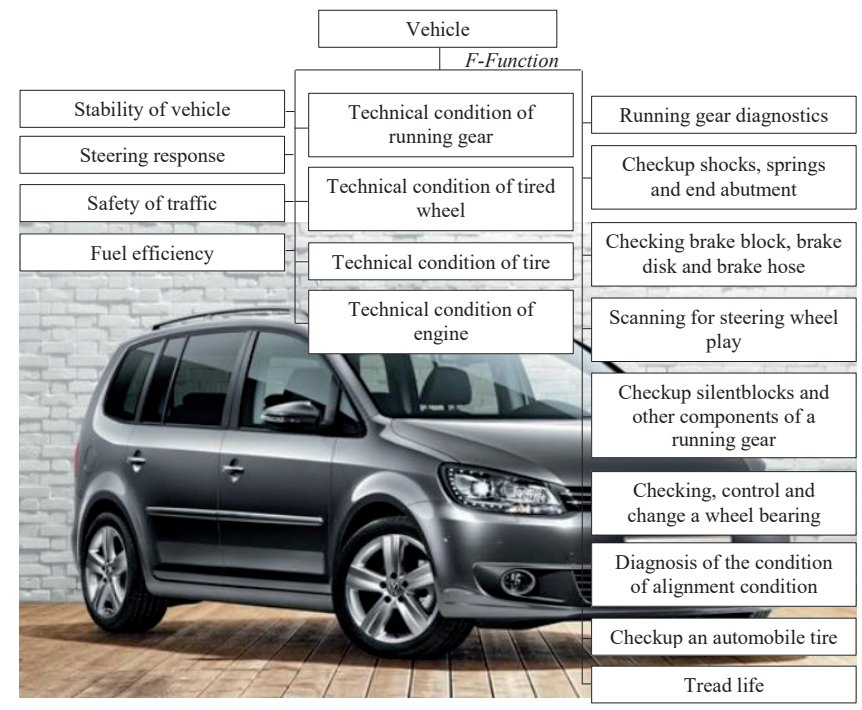

Figure 3: Function of a car for the FRAM. 
and steering tips, silent blocks; adjustment and replacement of wheel bearings; checking the wheel alignment; checking the condition of the car tire and monitoring its resource (Fig. 2). All this affects the operational properties of vehicles during their activity: stability, controllability, fuel efficiency. So, when the resource of a car tire approaches its limit state, the probability of a traffic accident increases [15]. In fig. 3 shows function of vehicle for FRAM. In fig. 4 (Table 2) shows a diagram of the chassis and the impact of its technical condition on the operational properties of a vehicle.

\section{Functionally-oriented technology in a vehicle maintenance system}

Functionally-oriented technology for conducting technical influences of vehicle is a special technology. This technology is based on an accurate topologically oriented implementation of the necessary set of algorithms for the technological influence of equipment and devices for vehicle maintenance. These actions are necessary for micro-, macro-zones and parts that are functionally consistent with their operating conditions. At the same time, their type, variant, quantity, quality and the algorithm of technological influence are purposefully determined, as well as topologically, functionally and quantitatively oriented during their realization into separate zones of part depending on the specified functional features of their operation. The use of function-oriented technologies for automobile components allows maximizing their overall operational parameters due to local increase in technical capabilities and properties of individual elements, surfaces and/or zones of a part, depending on the functional features of their operation [16].

Thus, it is necessary to form a new system of vehicles maintenance [17-19], which defines modern principles and methods (Fig. 5). Its functioning to a greater extent will be able to ensure traffic safety when executing various transporttechnological schemes for the delivery of cargo and transportation of passenger. This simulation system of a qualitatively new technology for technical maintenance of vehicles is implemented in the shell of a system of a qualitatively new technology for designing vehicles of a new generation.

The system of principles and methods for providing the qualitatively new properties of the maintenance technologies of new generation vehicles can be formed within the limits of a recurrence approach (repeatability) based on the following types of principles:

a) Well-known principles that can be represented by a set $R_{s}=\left\{R_{s 1}, R_{s 2}, R_{s 3}, \ldots, R_{s i}, . . R_{s p}\right\}$;

b) New principles $R_{n}=\left\{R_{S n 1}, R_{n 2}, R_{n 3}, \ldots, R_{n i}, \ldots R_{n j}\right\}$

c) Qualitatively new principles $R_{h}=\left\{R_{h 1}, R_{h 2}, R_{h 3}, \ldots, R_{h i}, . . R_{h g}\right\}$;

d) Fundamental new principles $R_{m}=\left\{R_{m 1}, R_{m 2}, R_{m 3}, \ldots, R_{m i}, . . R_{m d}\right\}$;

e) Principles at the level of new solutions

$R_{x}=\left\{R_{x 1}, R_{x 2}, R_{x 3}, \ldots, R_{x i}, . . R_{x v}\right\}$ and

f) $\ldots$;

Principles at the level of discovery

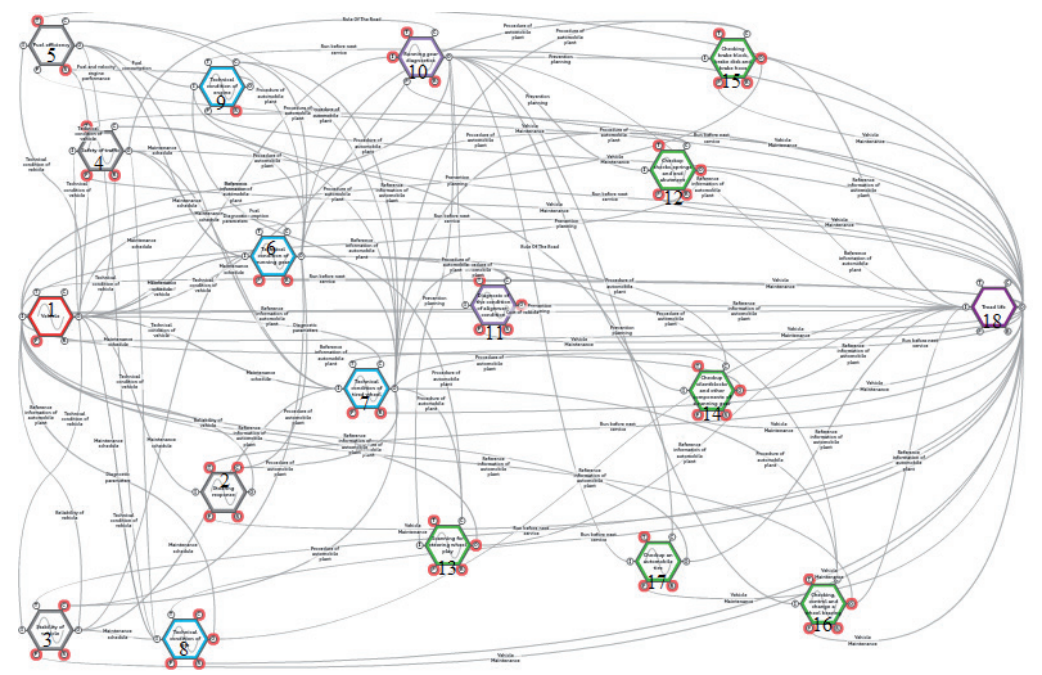

Figure 4: Functional Resonance Analysis Method simulation of the traffic safety control of the vehicle due to changes in its technical condition during operation. 
$R_{w}=\left\{R_{w 1}, R_{w 2}, R_{w 3}, \ldots, R_{w i}, \ldots R_{w y}\right\}$.

The system of principles and methods for providing qualitatively new properties and capabilities of the maintenance technology of new generation vehicles can also be formed within the limits of the generation of a set of variants of compositions of such principles on the basis of the morphological box:

$$
R=\left\|\begin{array}{|lllllll}
R_{s 1} & R_{s 2} & R_{s 3} & \ldots & R_{s i} & \ldots & R_{s p} \\
R_{n 1} & R_{n 2} & R_{n 3} & \ldots & R_{n i} & \ldots & R_{n j} \\
R_{h 1} & R_{h 2} & R_{h 3} & \ldots & R_{h i} & \ldots & R_{h g} \\
R_{m 1} & R_{m 2} & R_{m 3} & \ldots & R_{m i} & \ldots & R_{m d} \\
R_{x 1} & R_{x 2} & R_{x 3} & \ldots & R_{x i} & \ldots & R_{x v} \\
\ldots & \ldots & \ldots & \ldots & \ldots & \ldots & \ldots \\
R_{w 1} & R_{w 2} & R_{w 3} & \ldots & R_{w i} & \ldots & R_{w y}
\end{array}\right\|
$$

Where $R$ is the general system of principles and methods for providing qualitatively new properties and capabilities of the new generation maintenance technology; $R_{i j}$ is the $i$-th principle of the $j$-th type of principles for providing qualitatively new properties and capabilities of the new generation maintenance technology.

It should be noted that any principle of creating new generation technologies $R_{n j}$ can be formed from the intersection of a certain subset of known $p_{i}$ principles: It should be noted that any principle of creating new generation technologies $R_{n j}$ can be formed from the intersection of a certain subset of known $p_{i}$ principles:

$$
R_{n j}=\bigcap_{i=1}^{p_{j}} p_{i}
$$

Where $R_{n j}$ is a field that forms the new principles of technology; $R_{s i}$ is many well-known principles of simulation of technology; $p_{i}$ is power of subset of known principles.

\section{Conclusions}

Using the method of functional resonance analysis to simulate the influence of the technical condition of the chassis of a vehicle on its operational properties showed that one of the important elements for the effective implementation of the transport-technological transportation process is a serviceable technical condition of vehicles, which carries out delivery of cargo and transportation of passenger. High-quality maintenance of vehicles, taking into account modern approaches to new generation technologies, ensures the safety of the transportation process. Simulation the influence

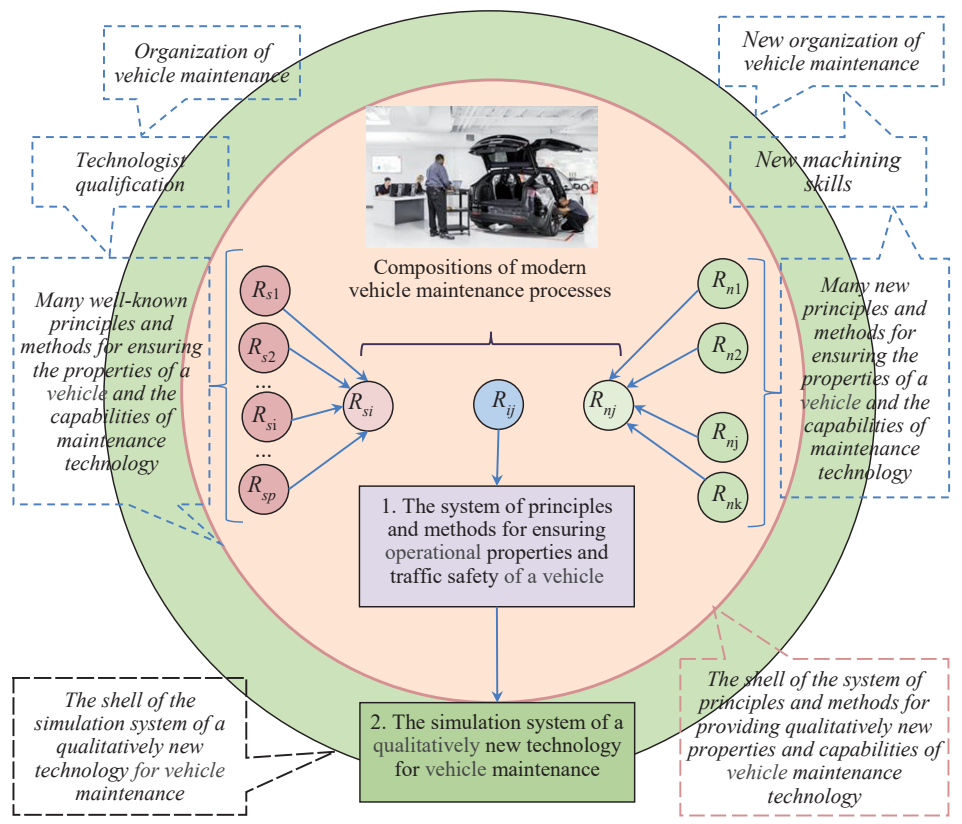

Figure 5: Hypothetical diagram of a general methodology for simulation a qualitatively new technology for maintenance and repair of vehicles (next-generation technology and new system "Person-Car-Organization of maintenance and repair system"). 
of the technical condition of the chassis on the service properties of a vehicles using method of functional resonance analysis clearly revealed that its maintenance is a complex type of human activity and requires new approaches to new generation technologies (new generation vehicles are hybrids, electric, autonomous). The FRAM model gave an idea of how successful and high-quality maintenance of vehicles depends on the complex interaction of several of its components working in the technical system. The interconnections are so complex that they are almost impossible to apprehend. Thus, the important socio-technical mechanisms of the "Person-Car-Organization of a maintenance and repair system" system regulate the communication between the functions of this system. They can be:

a) The informal control mechanisms;

b) The human control mechanisms;

c) A new generation vehicle maintenance technologies or specific tools such as monitoring the technical condition of the main units of vehicles;

d) The technological process of vehicle maintenance and diagnostics (both general and element-wise) and

e) The instructions and regulations for the repair team workers and similar documents.

Simulation the influence of the technical condition of the chassis of a vehicle on its operational properties by the method of functional resonance analysis showed that it is necessary to pay special attention to modern element-by-element diagnostics, since this significantly affects traffic safety. The fulfilment of these functions is based more on modern knowledge about the design of new generation vehicles and their maintenance technology.

The method of analysis of functional resonance complements the approach to solving the issues of efficiency of transportation process from the point of view of ensuring traffic safety of vehicles during it execution, improving the operational properties of vehicles. It is an innovative method that finds its application in medicine, nuclear energy, and the transport sector. This method is used to analyse the daily activities of transport enterprises in order to create models for specific tasks. The model of the FRAM can be used for specific types of analysis in order to verify the feasibility of the proposed solutions or changes to increase the efficiency of transport enterprises activity, to identify the causes of possible errors or threats, to understand the stages of the implementation and functioning of transport-technological processes of cargo delivery and transportation of passengers. It can be used as a basis for analysing the risks of traffic accidents and investigating them, or forecasting possible future events.

The use of function-oriented technologies in a vehicle maintenance system allows simulation and maximizing overall operational parameters of vehicle due to local increase in technical capabilities and properties of individual elements, surfaces and/ or zones of a part, depending on the functional features of operation.

\section{Acknowledgments}

The authors would like to express their gratitude for helping to leading scientists Professor DSc. Alexander Mihaylov (Donetsk National Technical University) and Erik Hollnagel, Ph.D., Professor, Professor Emeritus.

\section{References and Notes}

1. Hollnagel, E. (2008). From FRAM (Functional Resonance Analysis Model) to FRAM (Functional Resonance Analysis Method) Presentation at the FRAM workshop. École des Mines de Paris - Centre for Research on Risk and Crises (CRC). http://functionalresonance.com/onewebmedia/ FRAMily_2008_Hollnagel.pdf

2. Braithwaite, J., Wears, R. L., Hollnagel, E. (2015). Resilient health care: turning patient safety on its head. International Journal for Quality in Health Care, 27 (5), 418-420, https:// doi.org/10.1093/intqhc/mzv063

3. Anderson, J. E., Kodate, N. (2015). Learning from patient safety incidents in incident review meetings: organisational factors and indicators of analytic process effectiveness. Safety Science, 809, 105-114.

4. Clay-Williams, R., Hounsgaard, J., Hollnagel, E. (2015). Where the rubber meets the road: using FRAM to align work-asimagined with work-as-done when implementing clinical guidelines. Implementation Science., 10 (1), 125.

5. Pickup, L., Atkinson, S., Hollnagel, E. and et al. (2017). Blood sampling - two sides to the story. Applied Ergonomics, 59, 234-242.

6. Raben, D.C., Bogh, S.B., Viskum, B. et al. (2017). Proposing leading indicators for blood sampling: application of a method based on the principles of resilient healthcare. Cognition, Technology \& Work, 19 (4), 809-817.

7. Belmonte, F., Schön, W., Heurley, L., Capel, R. (2011). Interdisciplinary safety analysis of complex sociotechnological systems based on the Functional Resonance Accident Model: an application to railway traffic supervision. Reliability Engineering and System Safety, 96, 237-249. 
8. ISO/IEC/IEEE 12207:2017 (2017). Systems and software engineering - Software life cycle processes.

9. Марьин, С. Л. (2000). Компьютерные технологии для проектирования и производства сложных изделий машиностроения. САПР и графика, 7, 41-45. [in Russian: Maryin, S. L. (2000). Computer technology for the design and manufacture of complex engineering products, CAD and Graphics, 7, 41-45].

10. Stupnytskyy, V. (2012). Use of the CAF-system (Computer Aided Forming) in Integer Computer Aided Manufacturing. Papers of the XX Ukrainian-Polish Conference on CAD in Machinery Design. Implementation and Educational Issues, 45-48.

11. Herrera, I. A., Woltjer, R. (2010). Comparing a multi-linear (STEP) and systemic (FRAM) method for accident analysis. Reliability Engineering and System Safety, 95, 1269-1275. https://econpapers.repec.org/article/eeereensy/v_3a95_3 ay_3a2010_3ai_3a12_3ap_3a1269-1275.htm

12. Rutkowska, P., Krzyżanowski, M. (2018). FRAM modelling of the transfer of control over aircraft. Scientific Journal of Silesian University of Technology. Series Transport, 101, 159166.

13. Macchi, L. (2010). A Resilience Engineering approach to the evaluation of performance variability: Development and application of the Functional Resonance Analysis Method for Air Traffic Management safety assessment. PhD Thesis, MINES ParisTech, France.

14. Davis, J. P., Hall, J. W. (2003). A software-supported process for assembling evidence and handling uncertainty in decision-making. Decision Support Systems, 35 (3), 415433.

15. Sakno O. P. (2013). Managing resources tires of transport by improving monitoring tread wear [Text]: Dis. ... candidate. Sc. Sciences: 05.22.20 Lugansk, 216 (in Ukrainian).

16. Михайлов, А. Н. (2009). Основы синтеза функциональноориентированных технологий машиностроения [in Russian: Mihaylov, A. N. (2009). Basics of the synthesis of function-oriented engineering technologies. Mashinostroenie, Moscow].

17. Сакно, О. П., Колеснікова, Т. М., Олло, В. П. (2019). Моделювання системи технології технічних впливів на автомобіль. Вісник Придніпровської державної академії будівництва та архітектури, 1 (249-250), 102-109. [in Ukrainian: Sakno, O. P., Kolesnikova, T. M., Ollo, V. P. (2019). Simulation of the system of the technology of technical influences on vehicle. Bulletin of Prydniprovs'ka State Academy of Civil Engineering and Architecture, 1 (249-250), 102-109]. DOI: 10.30838/J.BPSACEA.2312.260319.102.412

18. Tian, W., Caponecchi, C. (2020). Using the Functional Resonance Analysis Method (FRAM) in Aviation Safety: A
Systematic Review. Journal of advanced transportation, October 2020, 14 pages. DOI: 10.1155/2020/8898903

19. Qiao, W., Li, X., Liu, Q. (2019). Systemic approaches to incident analysis in coal mines: comparison of the STAMP, FRAM and "2-4" Models. Resources Policy, 63. 